Check for updates

Cite this: RSC Adv., 2018, 8, 22748

\title{
One-pot synthesis of enhanced fluorescent copper nanoclusters encapsulated in metal-organic frameworks $\dagger$
}

\author{
Bingyan Han, Xixi Hu, Mingbo Yu, Tingting Peng, Ying Li and Gaohong He (D)*
}

The encapsulation of Cu nanoclusters (Cu NCs) in metal-organic frameworks (MOFs) would improve the properties of $\mathrm{Cu}$ NCs. So far, these composites were reported by a two-step synthesis process. In this work, a facile one-pot synthesis of hybridization of glutathione (GSH) protected Cu NCs (Cu NCs@GSH) and MOF-5 (Cu NCs@GSH/MOFs) composites was reported for the first time. The results of UV-vis, TEM, XPS and SEM proved Cu NCs@GSH were distributed homogeneously over the entire MOF structure. The fluorescence intensity of $\mathrm{Cu}$ NCs encapsulated in MOF-5 was enhanced about 35-fold owing to the confining scaffold of the MOF and the stability was extended from 3 days to 3 months. Cu NCsaGSH/ MOFs composites exhibited strong orange fluorescence and the emissions could change between blue, orange and red as they were partially reversible in different $\mathrm{pH}$ environments. This one-pot synthetic strategy could be extended for the encapsulation of fluorescent Ag NCs in MOFs as well. As-prepared Cu NCs@GSH/MOF-5 composites had high stability, and were easily recycled by centrifugation in aqueous solution, therefore, it would be utilized to develop a reusable sensor for detection of metal ions in the future.

Received 27th April 2018

Accepted 12th June 2018

DOI: $10.1039 / c 8 r a 03632 b$

rsc.li/rsc-advances

\section{Introduction}

Metal nanoclusters (NCs) have attracted tremendous attention as the emerging nanomaterials. Composed of several to dozens of metal atoms, their size is less than the Fermi wavelength of an electron. ${ }^{1}$ Owing to the strong quantum confinement of free electrons in this size regime, they exhibit excellent chemical, physical and optical properties. Photoluminescence (PL) in UV and visible regions is one of the most amazing properties compared to larger metal nanoparticles. Besides noble metal Au and $\mathrm{Ag}$, cheap, non-toxicity and abundant $\mathrm{Cu}$ has also been extensively studied..$^{2-4}$ However, because of susceptibility to oxidation and difficulty in size-control in the process of synthesis, poor stability and low fluorescence quantum yield (QY) are the major challenges of $\mathrm{Cu}$ NCs, which have retarded their practical applications and stimulated researchers to develop more simple and effective protocols to prepare Cu NCs. To date, much effort has been devoted to improving these two properties of $\mathrm{Cu}$ NCs by optimizing reaction conditions and/or selecting appropriate surface ligands. There have been lots of reports based on this idea involving the optimization of temperature, ${ }^{5} \mathrm{pH}$ values, ${ }^{6}$ concentration of reactants ${ }^{7,8}$ and the

State Key Laboratory of Fine Chemicals, School of Petroleum and Chemical Engineering, Dalian University of Technology, Panjin, Liaoning 124221, China. E-mail: hgaohong@dlut.edu.cn; Tel: +86427-2631809

$\dagger$ Electronic supplementary information (ESI) available. See DOI: 10.1039/c8ra03632b choice of proper reducing agents. For example, our group just utilized the hydroxylamine hydrochloride as reducing agent instead of hydrazine hydrate to synthesize ultrastable orangered-emitting $\mathrm{Cu}$ NCs for the first time. ${ }^{9}$ The use of relatively large size capping agents, or special reaction condition, such as microwave assisted, ${ }^{\mathbf{1 0}}$ or sonochemical synthesis, ${ }^{\mathbf{1 1}}$ was more conducive to the formation of high-performance $\mathrm{Cu}$ NCs. In addition, the strategy of the aggregation-induced emission (AIE), ${ }^{12}$ and the charge repulsion ${ }^{13}$ also have been extended to improve the optical properties of $\mathrm{Cu}$ NCs.

Metal-organic frameworks (MOFs) are a novel class host of highly porous materials constructed from metal ions and organic linkers. Four well-established strategies have been exploited for encapsulating dyes, ${ }^{\mathbf{1 4}, 15}$ polymers, ${ }^{\mathbf{1 6}}$ biomolecules, ${ }^{17}$ particularly nanomaterials (NMs) within MOFs according to the order of the growth of the materials: ship-in-bottle strategy (assembly of NCs inside MOFs), bottle-around-ship strategy (assembly of MOFs around NCs), sandwich assembly strategy (embedding NCs between MOFs layers) and in situ encapsulation (simultaneous synthesis of MOFs and NCs). ${ }^{18}$ The resulting composites enhanced performance characteristics of the guest materials in many fields such as gas storage, ${ }^{19,20}$ catalysis, ${ }^{21}$ and sensors. ${ }^{22}$ Nevertheless, there are only few reports about the encapsulation of fluorescent metal NCs into MOFs to enhance the fluorescence intensity and stability of NCs taking advantages of tunable porosity of MOFs. The combination of $\mathrm{Au}$ NCs and zeolitic-imidazolate frameworks-8 (ZIF-8) was applied for the first time in real-time monitoring of drug 
release. ${ }^{23}$ The Ag NCs encapsulated in MOFs exhibited enhanced catalytic activity towards the hydrogenation of 4nitroaniline. ${ }^{24}$ Wang et al. improved both the stability and the emission intensity of $\mathrm{Cu}$ NCs based on their confinementassisted effect of ZIFs. The emission intensity enhanced 20 times and maintained stable after two weeks compared to several hours. ${ }^{25}$ However, this method belonged to bottlearound-ship strategy (assembly of ZIFs around NCs) and it involved a two-step synthesis processes: at first $\mathrm{Cu}$ NCs cores with uniform sizes, shapes, and structures were prepared, and then ZIFs were assembled on the $\mathrm{Cu}$ NCs surfaces. In order to save time and reduce costs, it is even a challenge for the fabrication of $\mathrm{Cu}$ NCs/MOFs composites by in situ encapsulation strategy. Because the NCs and MOFs should be formed under the same reaction conditions in this method, synchronous control of the nucleation and growth of these two distinct components was not easy.

Herein, glutathione (GSH) protected Cu NCs (Cu NCs@GSH) and metal-organic frameworks-5 (MOF-5) were produced simultaneously, and the $\mathrm{Cu}$ NCs were encapsulated in MOF-5 shells by a facile one-pot synthesis at room temperature. The fluorescence intensity has been enhanced about 35 folds and the stability prolonged from 3 days to at least 3 months by making use the cavities of the rigid confining scaffold of MOF-5 to stabilize Cu NCs@GSH. Moreover, the Cu NCs@GSH/MOF-5 composites were sensitive to the change of $\mathrm{pH}$ and the fluorescence emissions were changed between blue, orange and red reversibly. To the best of our knowledge, it is the first time to obtain hybrid material of Cu NCs@GSH/MOF-5 by one-pot synthesis. Moreover, this idea was also successfully extended to the mode of Ag NCs and MOF-5, where the emission of Ag NCs was improved as well.

\section{Experimental section}

\section{Materials}

Copper nitrate trihydrate $\left[\mathrm{Cu}\left(\mathrm{NO}_{3}\right)_{2} \cdot 3 \mathrm{H}_{2} \mathrm{O}\right]$, 1,4-benzenedicarboxylic acid $\left(\mathrm{H}_{2} \mathrm{BDC}\right)$ and $\mathrm{GSH}$ were purchased from Sangon Biotechnology Co. Ltd (Shanghai, China). Zinc nitrate hexahydrate $\left[\mathrm{Zn}\left(\mathrm{NO}_{3}\right)_{2} \cdot 6 \mathrm{H}_{2} \mathrm{O}\right]$ was ordered from STREM CHEMICALS, INC. $N, N$-dimethylformamide (DMF) was bought from Damao Chemical Reagent Co., Ltd (Tianjin, China). Sodium hydroxide $(\mathrm{NaOH})$ was acquired from Tianli Chemical Reagent Co., Ltd (Tianjin, China).

\section{Instrumentation}

The UV-visible spectra were recorded using a UV-Vis Cary 60 spectrophotometer at room temperature (Agilent, China). The fluorescence spectra were studied using Hitachi F-7000 fluorescence spectrophotometer (Tokyo, Japan). X-ray photoelectron spectroscopy (XPS) measurements were performed by using a VG Thermo ESCALAB 250 spectrometer. The transmission electron microscope (TEM) images were acquired on a Tecnai G2 F30 S-TWIN. The scanning electron microscope (SEM) was carried out on Nova 450 Nano SEM. X-ray diffraction (XRD) measurements were performed via a Shimadzu 7000S diffract meter using $\mathrm{Cu} \mathrm{K} \alpha$ radiation. The zetapotential was performed on Zetasizer Nano-ZEN3700 instrument (Malvern, UK).

\section{Synthesis of MOF-5}

MOF-5 was synthesized according to the literature with some modifications. ${ }^{25} 0.831 \mathrm{~g} \mathrm{Zn}\left(\mathrm{NO}_{3}\right)_{2} \cdot 6 \mathrm{H}_{2} \mathrm{O}$ and $0.178 \mathrm{~g} \mathrm{H} \mathrm{H}_{2} \mathrm{BDC}$ were dissolved in $20 \mathrm{~mL}$ DMF under stirring until the complete dissolution. Then the mixture was transferred into reaction still for $4 \mathrm{~h}$ at $130{ }^{\circ} \mathrm{C}$. White crystals were obtained after cooled to room temperature and filtration. Then the crystals were washed by DMF for several times and dried at $60^{\circ} \mathrm{C}$ for $12 \mathrm{~h}$ for further use.

\section{Synthesis of Cu NCs@GSH}

The preparation of $\mathrm{Cu}$ NCs@GSH was according to the literature of Luo et al. $^{7}$ with some modifications. In a typical experiment, $5 \mathrm{~mL} 50 \mathrm{mg} \mathrm{mL}{ }^{-1} \mathrm{GSH}$ solution was added into $5 \mathrm{~mL}$ $10 \mathrm{mM} \mathrm{Cu}\left(\mathrm{NO}_{3}\right)_{2}$ solution under stirring. Then, $2 \mathrm{M} \mathrm{NaOH}$ solution was used to adjust the $\mathrm{pH}$ value to 5 . The mixture turned to be pale yellow transparent solution. After vigorously stirring for $4 \mathrm{~h}$ at $37^{\circ} \mathrm{C}$, as-prepared Cu NCs@GSH showed weak orange fluorescence when excited at $380 \mathrm{~nm}$.

\section{Synthesis of Cu NCs@GSH/MOF-5 composites}

The Cu NCs@GSH/MOF-5 composites were synthesized by onepot method. Typically, $0.831 \mathrm{~g} \mathrm{Zn}\left(\mathrm{NO}_{3}\right)_{2} \cdot 6 \mathrm{H}_{2} \mathrm{O}$ and $0.178 \mathrm{~g}$ $\mathrm{H}_{2}$ BDC were dissolved in $20 \mathrm{~mL}$ DMF under stirring. $2.5 \mathrm{~mL}$ $10 \mathrm{mM} \mathrm{Cu}\left(\mathrm{NO}_{3}\right)_{2} \cdot 3 \mathrm{H}_{2} \mathrm{O}$ solution and $2.5 \mathrm{~mL} 50 \mathrm{mM} \mathrm{GSH}$ solution were introduced into the above transparent solution. The mixture turned to white solution when the $\mathrm{pH}$ value was adjusted to 5 using $2 \mathrm{M} \mathrm{NaOH}$. White turbid solution was obtained after vigorously stirring for $24 \mathrm{~h}$ at room temperature. The products were stood and then the supernatant was removed. The white precipitate was washed by $5 \mathrm{~mL}$ DMF for 3 times. Subsequently, the white precipitate was dried at $60{ }^{\circ} \mathrm{C}$ for $12 \mathrm{~h}$, obtaining white powder products, which were stored at room temperature for further use and characterization.

\section{Results and discussion}

Fabrication process of enhanced fluorescent $\mathrm{Cu}$ NCs@GSH/ MOF-5 composites by one-pot strategy at room temperature for the first time is seen in Scheme 1. The individual $\mathrm{Cu}$

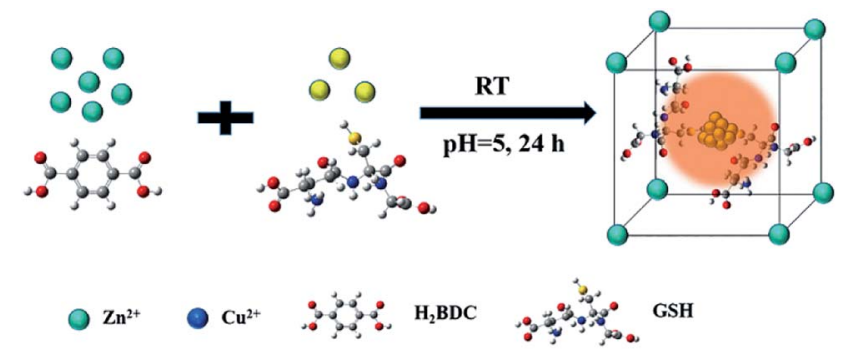

Scheme 1 Schematic illustration of the one-pot synthesis of $\mathrm{Cu}$ NCs@GSH/MOF-5 composites. 
NCs@GSH synthesized according to the reported method exhibit faint fluorescence at $600 \mathrm{~nm}$ when excited at $380 \mathrm{~nm}$, shown in Fig. 1a. However, Cu NCs@GSH encapsulated in MOF5 (Cu NCs@GSH/MOF-5) show strong orange emission at
$580 \mathrm{~nm}$ with the same excitation wavelength. The intensity is about 35 times than that of individual Cu NCs@GSH. We suspect that the Cu NCs@GSH are encapsulated in the pores of the MOF-5 structure, restricting the vibration of protecting (a)

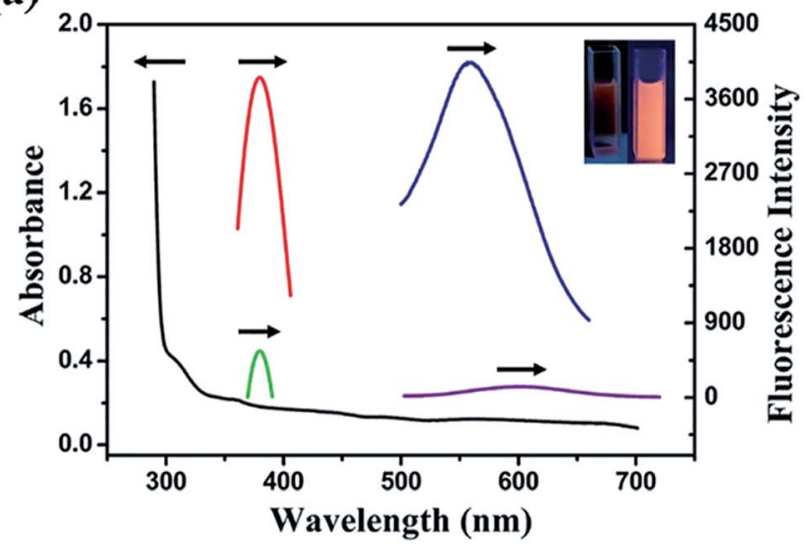

(b)

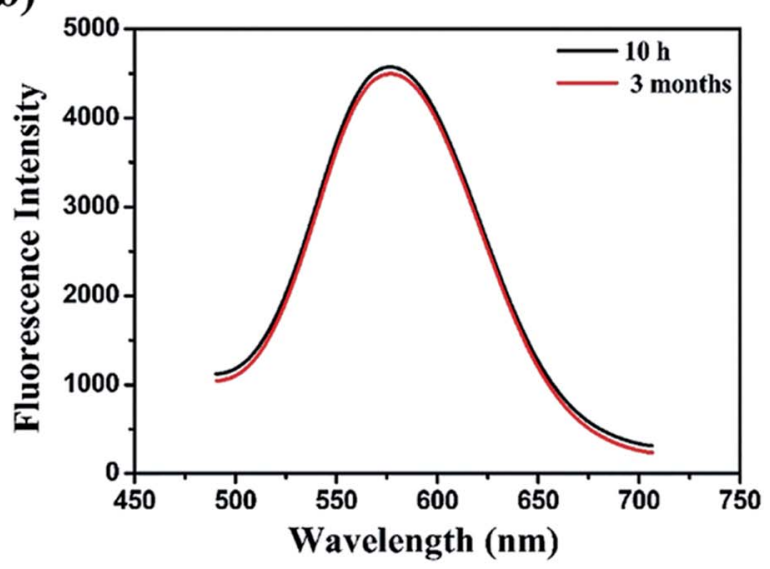

Fig. 1 (a) The absorption (black line), excitation (red line), emission (blue line) spectra of Cu NCsaGSH/MOF-5 composites and excitation (green line), emission (purple line) spectra of Cu NCs@GSH. Insets show the photographs of Cu NCs@GSH (left cuvette) and Cu NCs@GSH/MOF-5 composites (right cuvette) under the $365 \mathrm{~nm}$ UV light. (b) The fluorescence emission spectra of Cu NCs@GSH/MOF-5 composites at different time.

(a)

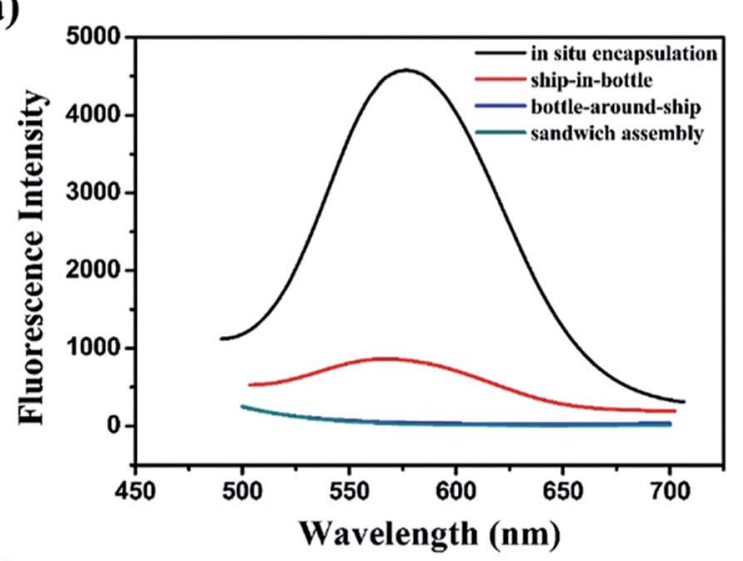

(c)

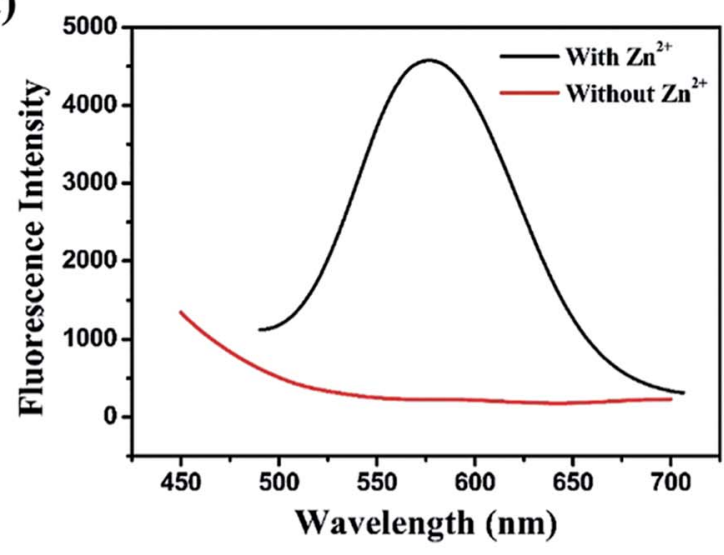

(b)

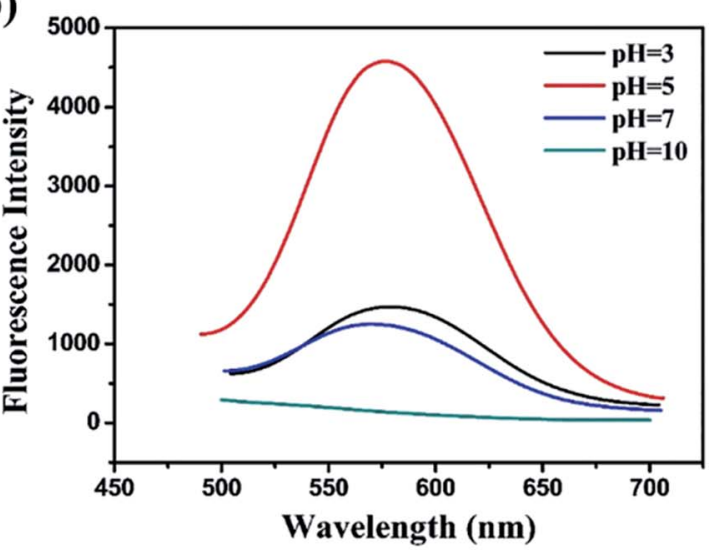

(d)

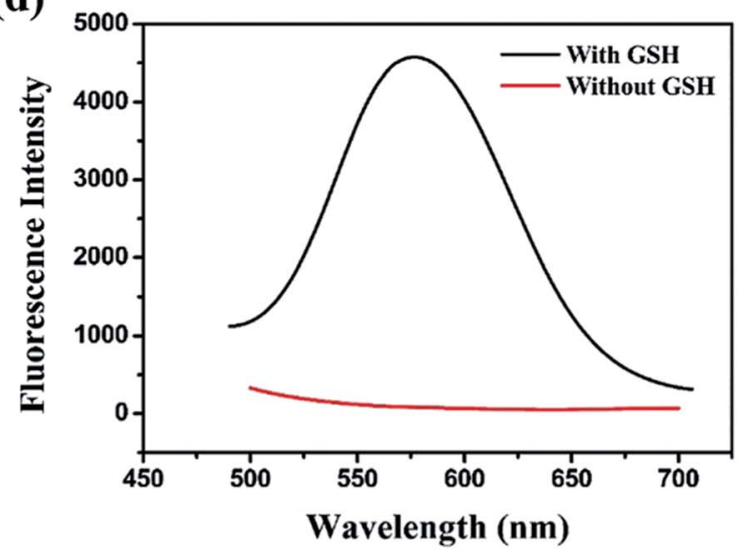

Fig. 2 (a) The impact of the addition sequence on the fluorescence intensity. (b) Fluorescence intensity of Cu NCs@GSH/MOF-5 composites at different $\mathrm{pH}$ values. (c) The difference of fluorescence intensity with and without $\mathrm{Zn}^{2+}$. (d) The difference of fluorescence intensity with and without GSH. 
groups on the surface of the Cu NCs@GSH, thus reduce the non-radiation transition. In addition, maybe the confined effect of MOF-5 lead to the predominance of inter- $\mathrm{Cu}^{1} \cdots \mathrm{Cu}^{1}$ interactions over intra-interactions, resulting in the blue shift of emission wavelength. ${ }^{26}$ The peak at around $350 \mathrm{~nm}$ of UV-vis spectrum, shown in Fig. 1a, is attributed to the interband electronic transition of the $\mathrm{Cu}$ NCs. The inexistence of absorption peak at 500-600 $\mathrm{nm}$ indicate that $\mathrm{Cu}$ NCs, rather than $\mathrm{Cu}$ nanoparticles, are encapsulated in MOF-5 successfully. The MOF-5 protects $\mathrm{Cu}$ NCs@GSH from being oxidized effectively, thus the stability is extended from 3 days to 3 months (Fig. 1b).

It is well known that the sequence of addition of reagents influences the experimental results obviously. Our strategy involves mixing all the necessary constituents at the same time, so it can be attributed to be "in situ encapsulation". Another three strategies, namely ship-in-bottle, bottle-around-ship and sandwich assembly are performed as well. The "ship-in-bottle" involves the formation of Cu NCs@GSH in the MOFs matrix that have already been formed. On the contrary, the "bottle-aroundship" is to guide the MOF-5 to be assembled around the preprepared Cu NCs@GSH. The last one, sandwich assembly, is individual preparation of MOFs and Cu NCs@GSH and subsequent mixing. As shown in Fig. 2a, the products prepared by the "ship-in-bottle" way exhibit weak orange emission at $580 \mathrm{~nm}$ and the latter two show hardly any fluorescence. Because the $\mathrm{Cu}$
NCs@GSH and MOF-5 must grow under the same reaction conditions, we speculate that $\mathrm{pH}$ value is another significant factor in the synthesis process. As shown in Fig. 2b, the intensity of fluorescence reveals obvious difference when the $\mathrm{pH}$ values of solution vary at 3, 5, 7 and 10 . The fluorescence intensity at pH 5 is about 3 times stronger than others. Theoretically, the alkaline environment is in favor of the crystallization of MOF-5 since it can accelerate the deprotonation of $\mathrm{H}_{2} \mathrm{BDC}$, and promote the coordination between $\mathrm{H}_{2} \mathrm{BDC}$ and $\mathrm{Zn}^{2+}$ to form MOF-5 structure. However, when the $\mathrm{pH}$ value is higher than 5 , it will severely disrupt the nucleation of Cu NCs@GSH according to the reported literature. ${ }^{6}$ Considering the intensity of fluorescence of the resulting composites, we chose the pH 5 as the optimum value. In addition, some control experiments have been carried out to confirm the successful synthesis of $\mathrm{Cu}$ NCs@GSH/MOF-5 composites. $\mathrm{Zn}^{2+}$ is essential for the formation of MOF-5. The mixture in the absence of $\mathrm{Zn}^{2+}$ is reacted at room temperature for $24 \mathrm{~h}$ and purified by the same procedure of the Cu NCs@GSH/MOF-5 composites. As shown in Fig. 2c, the fluorescence intensity without $\mathrm{Zn}^{2+}$ is much lower than that with $\mathrm{Zn}^{2+}$. And the decrease of amount of $\mathrm{Zn}^{2+}$ will result in the reduced fluorescence intensity of $\mathrm{Cu}$ NCs@GSH/MOF-5 composites dramatically, shown in Fig. S1. $\dagger$ The mentioned above prove effectively that the $\mathrm{Zn}^{2+}$ in the MOF-5 plays an important role for the enhancement of the optical properties of (a)

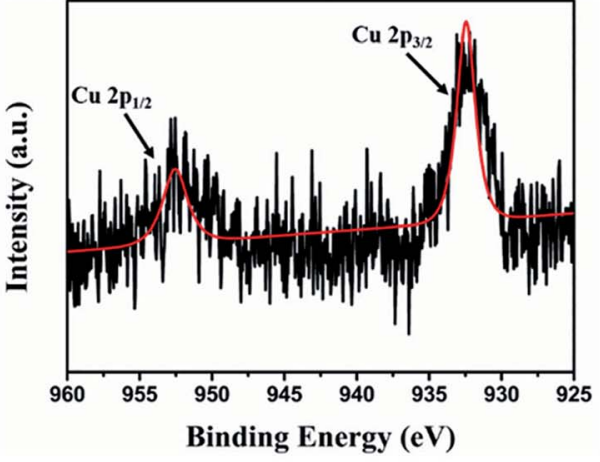

(c)
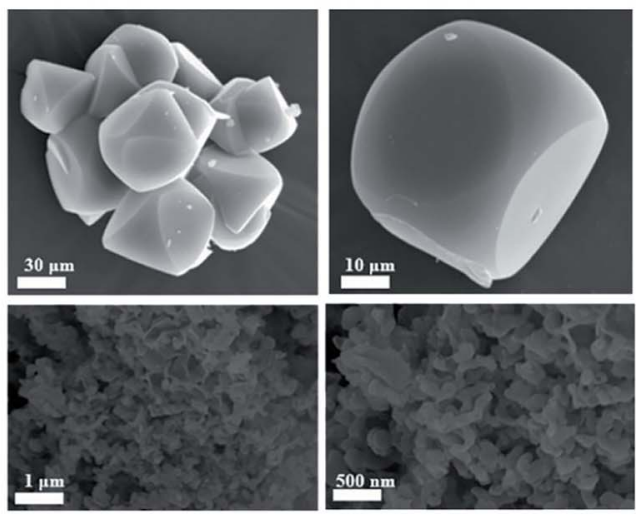

(b)
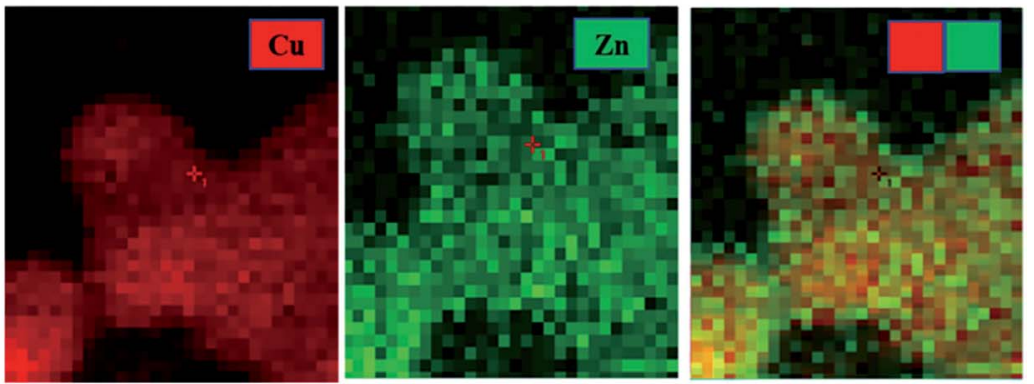

(d)

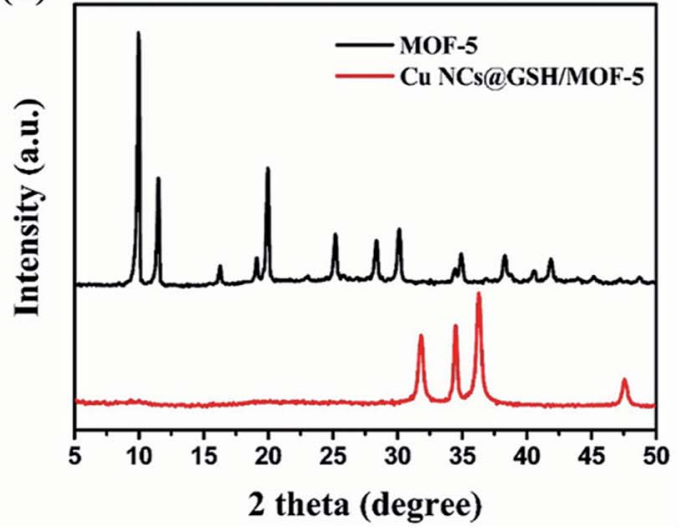

Fig. 3 (a) XPS spectrum of Cu 2p of Cu NCs@GSH/MOF-5 composites. (b) EDS elemental mapping of Cu, Zn and overlapped EDS images of Cu NCs@GSH/MOF-5 composites. (c) SEM images of MOF-5 (upper) and Cu NCs@GSH/MOF-5 composites (lower). (d) XRD diffraction patterns of MOF-5 and Cu NCs@GSH/MOF-5 composites. 
the Cu NCs@GSH. The addition of $\mathrm{Zn}^{2+}$ not only can enhance the fluorescence intensity of Cu NCs@GSH/MOF-5 but results in the blue shift of emission wavelength compared with that of $\mathrm{Cu}$ NCs@GSH. We speculate that $\mathrm{Zn}^{2+}$ has synergetic effect with $\mathrm{Cu}^{2+}$ on the synthesis of $\mathrm{Cu}$ NCs@GSH/MOF-5 to some extent. Similarly, in the absence of GSH, as shown in Fig. 2d, there is nearly no fluorescence emission as well. This result illustrates that the capping agent GSH, which can prevent $\mathrm{Cu}$ NCs aggregating into larger non-fluorescent nanoparticles, plays a key role in the preparation of fluorescent Cu NCs of the Cu NCs@GSH/ MOF-5. The role of each component on the formation of $\mathrm{Cu}$ NCs@GSH/MOF-5 have been investigated in more detail, seen in Table S1, Fig. S2 and S3. $\dagger$

XPS spectrum showed the peaks at $932 \mathrm{eV}$ and $952 \mathrm{eV}$ can be assigned to $\mathrm{Cu}_{2 \mathrm{p} 3 / 2}$ and $\mathrm{Cu}_{2 \mathrm{p} 1 / 2}$, which are the characteristic peaks of $\mathrm{Cu}^{0}$, as shown in Fig. 3a. No peak is observed at $942 \mathrm{eV}$, indicating that there is no $\mathrm{Cu}^{2+}$ in $\mathrm{Cu}$ NCs@GSH/MOF-5 composites. $^{27}$ As shown in Fig. 3b, EDS elemental mapping verifies the uniform distribution of $\mathrm{Cu}$ element and demonstrates that Cu NCs@GSH are successfully encapsulated in MOF5. This is further confirmed by perfectly matching the green and red colour corresponding to $\mathrm{Zn}$ and $\mathrm{Cu}$ elements shown in an overlapped images. $\mathrm{Zn}^{2+}$ and $\mathrm{Cu}^{2+}$ are distributed uniformly in the Cu NCs@GSH/MOFs and the EDS elemental mapping mainly indicates the qualitative analysis of $\mathrm{Zn}^{2+}$ and $\mathrm{Cu}^{2+}$. As a result, there is no obvious difference in EDS elemental mapping. The specific amount of elementals in the Cu NCs@GSH/MOF-5 composites are showed in Fig. S4. $\dagger$ A series of results above mentioned indicate the successful synthesis of fluorescent $\mathrm{Cu}$ NCs@GSH encapsulated into MOF-5. The size and morphology of the as-prepared $\mathrm{Cu}$ NCs@GSH/MOF-5 composites are investigated by SEM and TEM, shown in Fig. 3c. The MOF- 5 consists of hexahedral structure and the diameter of individual crystal is about $10 \mu \mathrm{m}$. On the contrary, the $\mathrm{Cu}$ NCs@GSH/MOF-5 composites are composed of abundant randomly nanosheets. This obvious difference in morphology reveals that the introduction of $\mathrm{Cu}$ NCs@GSH influences the self-assembly of MOF-5. The Cu NCs@GSH are negatively charged with a zeta potential of $-14.6 \mathrm{mV}$. However, the MOF-5 is positively charged with abundant $\mathrm{Zn}^{2+}$. Therefore, maybe the electrostatic interaction drives Cu NCs@GSH being encapsulated in the pores of MOF-5 shells. Furthermore, TEM image of Cu NCs@GSH/MOF-5, showed in Fig. S5, $\uparrow$ exhibits great consistency with the images of SEM, which proves convincingly that the introduction of $\mathrm{Cu}$ NCs rather than temperature affects the morphology. The XRD patterns of MOF-5 and Cu NCs@GSH/MOF-5 composites indicate the growth of $\mathrm{Cu}$ NCs@GSH and MOF-5 is influenced each other dramatically, shown in Fig. 3d.

The original Cu NCs@GSH/MOF-5 composites are white turbid liquid with strong orange emission at $580 \mathrm{~nm}$ at $\mathrm{pH} 5$. But, the crystal structure of MOF-5 is vulnerable to be destructed in acid solution. ${ }^{28}$ Upon the addition of $\mathrm{HCl}$ solution, MOF-5 is collapsed, which results in the phenomenon that turbid liquid of orange $\mathrm{Cu}$ NCs@GSH/MOF-5 turns into transparent solution while the Cu NCs@GSH are exposed directly to the solution, as shown in route $\mathrm{H}^{+}(1)$ in Fig. $4 \mathrm{a}$. When the $\mathrm{pH}$ decreases about 3.5 , the solution exhibits blue emission at (a)

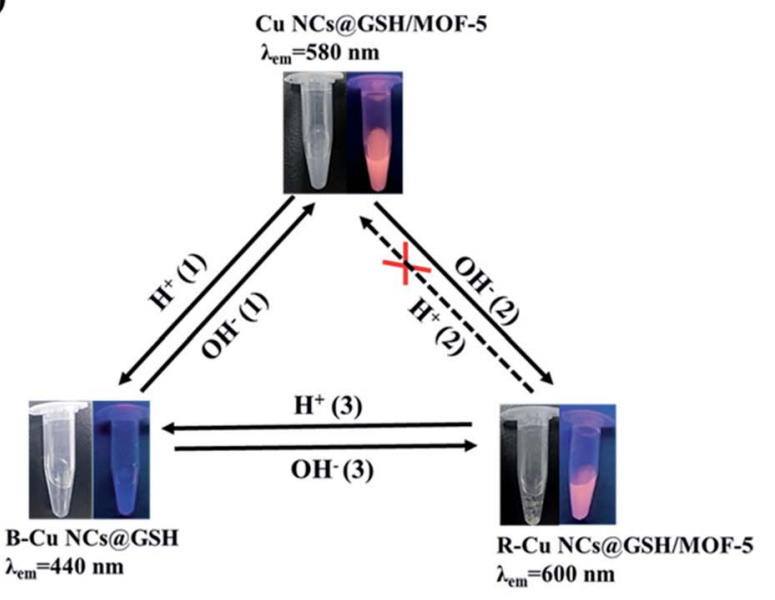

(b)

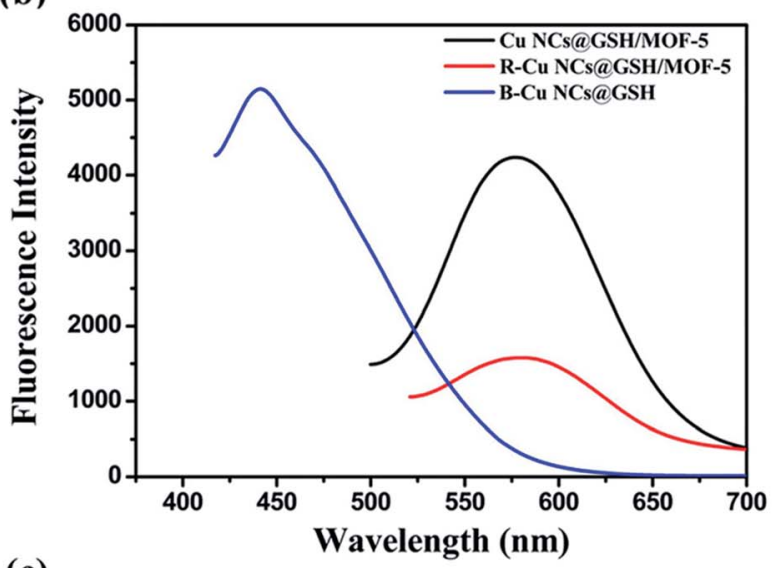

(c)
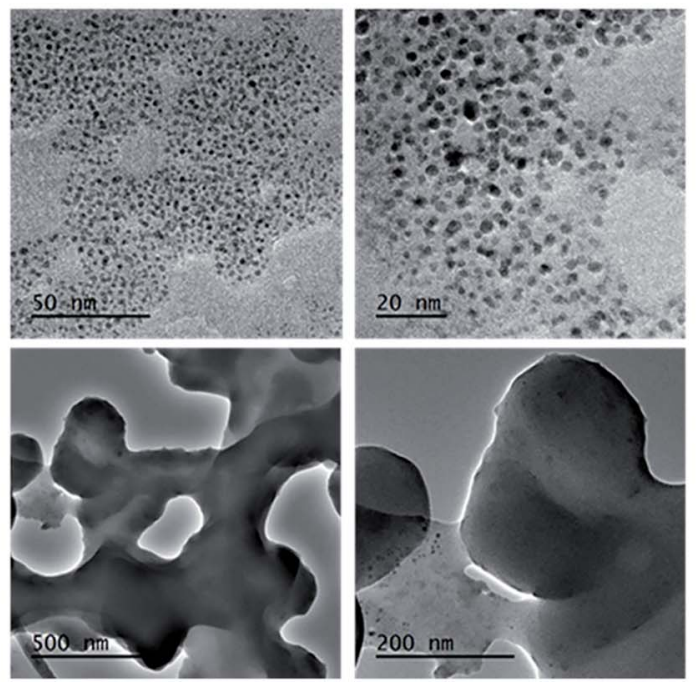

Fig. 4 (a) The inter-conversion of color of $\mathrm{Cu}$ NCsaGSH/MOF-5 composites upon the addition of $1 \mathrm{M} \mathrm{NaOH}$ and $1 \mathrm{M} \mathrm{HCl}$ solution. Photos show $\mathrm{Cu}$ NCs@GSH/MOF-5, B-Cu NCs@GSH and R-Cu NCs@GSH/MOF-5 under light (left) and UV light (right). (b) Fluorescence spectra of Cu NCs@GSH/MOF-5, R-Cu NCs@GSH/MOF-5 and B-Cu NCs@GSH. (c) TEM images of Cu NCs@GSH/MOF- 5 composites in acid or base solution (upper: $1 \mathrm{M} \mathrm{HCl}$ solution; lower: $1 \mathrm{M} \mathrm{NaOH}$ solution). 
$440 \mathrm{~nm}$. Moreover, the fluorescence intensity is enhanced about $25 \%$, shown in Fig. 4b. The TEM images reveal there is no structure of MOF-5 and the released Cu NCs@GSH show spherical morphology and good dispersibility (Fig. 4c upper). The average diameter of the Cu NCs@GSH is about $2.0 \mathrm{~nm}$ by calculating 100 particles. It is obvious that the $\mathrm{pH}$ leads to the blue shift of emission wavelength and enhancement of fluorescence intensity of $\mathrm{Cu}$ NCs@GSH. This solution is marked as blue-Cu NCs@GSH (B-Cu NCs@GSH). When some $\mathrm{NaOH}$ solution was added into B-Cu NCs@GSH, white precipitate reappeared. It indicated the MOF-5 can partially recovered, and orange emission reappeared, seen route $\mathrm{OH}^{-}$(1). If $\mathrm{NaOH}$ solution was excess, the solution becomes more turbid. It shows the structure of MOF-5 continued to grow and made the $\mathrm{Cu}$ NCs@GSH were much more tightly encapsulated, which lead to the decrease of fluorescence intensity and red shift of fluorescence emission wavelength, marked as red-Cu NCs@GSH/MOF5 (R-Cu NCs@GSH/MOF-5), as shown in route $\mathrm{OH}^{-}$(2). When $\mathrm{pH}$ increases to about 8.5, they show red emission at $600 \mathrm{~nm}$. It meant if $\mathrm{NaOH}$ solution was enough, B-Cu NCs@GSH can changed to R-Cu NCs@GSH/MOF-5 directly, as shown in route $\mathrm{OH}^{-}$(3). TEM images indicate that MOF-5 is still hybrided with $\mathrm{Cu}$ NCs@GSH in the process of addition of $1 \mathrm{M} \mathrm{NaOH}$ solution (Fig. 4c lower). Moreover, the SEM images show that $\mathrm{Cu}$ NCs@GSH/MOF-5 exhibit more distinct space structure (Fig. S6†), which is in complete agreement with the more generation of precipitate. However, because the MOF-5 is sensitive with acidic conditions, upon addition of $\mathrm{HCl}$, the structure of MOF-5 in the R-Cu NCs@GSH/MOF-5 would collapse completely to release the $\mathrm{Cu}$ NCs, and turn to $\mathrm{B}-\mathrm{Cu}$ NCs@GSH over the orange Cu NCs@GSH/MOF-5, as shown in route $\mathrm{H}^{+}(1)$ and (3). The dissolution rate of MOF-5 is so fast that the precise control of the appearance of orange fluorescence is difficult (route $\mathrm{H}^{+}$(2)). In short, $\mathrm{Cu}$ NCs@GSH/MOF-5 composites can achieve the inter-conversion of color in acid or base solution, shown in Fig. 4 a.

It is important that this one-pot synthetic method can be also extended for the encapsulation of fluorescent Ag NCs in MOF-5 at room temperature owing to the similar properties with Cu. Similarly, the Ag NCs@GSH/MOF-5 composites exhibit strong yellow fluorescence under UV light, shown in Fig. S7a. $\dagger$ The peaks at $368.2 \mathrm{eV}$ and $374.2 \mathrm{eV}$ of XPS spectrum of Ag, shown in Fig. $S 7 b, \dagger$ are assigned to $\mathrm{Ag} 3 \mathrm{~d}_{5 / 2}$ and $3 \mathrm{~d}_{3 / 2}$, which indicates the existence of $\mathrm{Ag}^{0}$. The SEM images of a uniform rod-like shape show Ag NCs@GSH/MOF-5 composites have uniform rod-like shape, and the length and width of "stick-like" composites are about $537.9 \mathrm{~nm}$ and $81.94 \mathrm{~nm}$, respectively, shown in Fig. S7c. $\uparrow$ The almost identical XRD diffraction peaks between MOF-5 and Ag NCs@GSH/MOF-5 composites demonstrate the minor influence of incorporated $\mathrm{Ag}$ NCs on the crystal structure of MOF-5, seen Fig. S7d. $\dagger$

\section{Conclusions}

In summary, Cu NCs@GSH and MOF-5 grew in one-pot at the same time while $\mathrm{Cu}$ NCs@GSH were distributed homogeneously over the entire MOFs structure. The optical properties and stability of $\mathrm{Cu}$ NCs@GSH were improved greatly by the confinement effect of the three-dimensional cavities in the MOF-5. Cu NCs@GSH/MOF-5 displayed conspicuously three fluorescence emission colours depending on the $\mathrm{pH}$ values, and the change of fluorescence emission was partially reversible. Since Cu NCs@GSH/MOF-5 composites had high stability, and were easily recycled by centrifugal in aqueous solution, therefore, it would be utilized to develop a reusable sensor for detection of metal ions in the future.

\section{Conflicts of interest}

There are no conflicts to declare.

\section{Acknowledgements}

This work was supported by Dalian High Level Talent Innovation Support Programme (Grant No. 2015R043), the Fundamental Research Funds for the Central Universities (Grant No. DUT16LK09), the Open Funds of the State Key Laboratory of Electroanalytical Chemistry (Grant No. SKLEAC201608) and Changjiang Scholars Program (Grant No. T2012049).

\section{References}

1 D. Li, Z. Chen and X. Mei, Adv. Colloid Interface Sci., 2017, 250, 25-39.

2 Y. L. Xiaohui Gao, M. Liu, S. He and W. Chen, J. Mater. Chem. C, 2016, 3, 4050-4056.

3 G. Zhang, T. Xu, H. Du, Y. Qiao, X. Guo, L. Shi, Y. Zhang, S. Shuang, C. Dong and H. Ma, J. Mater. Chem. C, 2016, 4, 3540-3545.

4 D. Li, Z. Chen, Z. Wan, T. Yang, H. Wang and X. Mei, RSC Adv., 2016, 6, 34090-34095.

5 Y. E. Shi, S. Luo, X. Ji, F. Liu, X. Chen, Y. Huang, L. Dong and L. Wang, Dalton Trans., 2017, 46, 14251-14255.

6 L. Lin, Y. Hu, L. Zhang, Y. Huang and S. Zhao, Biosens. Bioelectron., 2017, 94, 523-529.

7 T. Luo, S. Zhang, Y. Wang, M. Wang, M. Liao and X. Kou, Luminescence, 2017, 32, 1092-1099.

8 B. Han, X. Hou, R. Xiang and G. He, Anal. Methods, 2017, 9, 4028-4032.

9 J. Xu and B. Han, Nano, 2016, 11, 1650108.

10 J. R. Bhamore, S. Jha, A. K. Mungara, R. K. Singhal, D. Sonkeshariya and S. K. Kailasa, Biosens. Bioelectron., 2016, 80, 243-248.

11 C. Wang, H. Cheng, Y. Huang, Z. Xu, H. Lin and C. Zhang, Analyst, 2015, 140, 5634-5639.

12 S. Yang, X. Sun and Y. Chen, Mater. Lett., 2017, 194, 5-8.

13 D. Li, Y. Zhao, Z. Chen, X. Mei and X. Qiu, Mater. Sci. Eng. C, 2017, 78, 653-657.

14 J. V. Morabito, L. Y. Chou, Z. Li, C. M. Manna, C. A. Petroff, R. J. Kyada, J. M. Palomba, J. A. Byers and C. K. Tsung, J. Am. Chem. Soc., 2014, 136, 12540-12543.

15 Y. Wen, T. Sheng, X. Zhu, C. Zhuo, S. Su, H. Li, S. Hu, Q. L. Zhu and X. Wu, Adv. Mater., 2017, 29, 1700778. 
16 T. Kitao, M. W. A. MacLean, B. Le Ouay, Y. Sasaki, M. Tsujimoto, S. Kitagawa and T. Uemura, Polym. Chem., 2017, 8, 5077-5081.

17 J. Zhuang, A. P. Young and C. K. Tsung, Small, 2017, 13, 1700880.

18 L. Chen, R. Luque and Y. Li, Chem. Soc. Rev., 2017, 46, 46144630.

19 J. Yu, L. H. Xie, J. R. Li, Y. Ma, J. M. Seminario and P. B. Balbuena, Chem. Rev., 2017, 117, 9674-9754.

20 Y. Guan, J. Shi, M. Xia, J. Zhang, Z. Pang, A. Marchetti, X. Wang, J. Cai and X. Kong, Appl. Surf. Sci., 2017, 423, 349-353.

21 F. G. Cirujano, I. Luz, M. Soukri, C. Van Goethem, I. F. J. Vankelecom, M. Lail and D. E. De Vos, Angew. Chem., 2017, 56, 13302-13306.
22 G. L. a. J. T. Hupp, J. Am. Chem. Soc., 2010, 132, 7832-7833. 23 F. Cao, E. Ju, C. Liu, W. Li and Y. Zhang, Nanoscale, 2017, 9, 4128-4134.

24 D. A. Islam, A. Chakraborty and H. Acharya, New J. Chem., 2016, 40, 6745-6751.

25 Z. Wang, R. Chen, Y. Xiong, K. Cepe, J. Schneider, R. Zboril, C.-S. Lee and A. L. Rogach, Part. Part. Syst. Charact., 2017, 34, 1700029.

26 J. Liu, Q. M. Zhang, Y. Feng, Z. Zhou and K. Shih, Chemphyschem, 2016, 17, 225-231.

27 X. Hu, T. Liu, Y. Zhuang, W. Wang, Y. Li, W. Fan and Y. Huang, TrAC, Trends Anal. Chem., 2016, 77, 66-75.

28 S. Han and M. S. Lah, Cryst. Growth Des., 2015, 15, 55685572 . 